\title{
DÉTECTION ACOUSTIQUE ACTIVE DE GAZ DANS UNE ENCEINTE MÉTALLIQUE
}

\author{
C. JOURNEAU \\ Commissariat à l'Energie Atomique, Centre de Cadarache, BP. 1, F-13108 St Paul lès Durance cedex, \\ France
}

A non intrusive process to detect the apparition of gas in a liquid phase contained in an elastic enclosure is described. A short duration acoustic wavetrain is emitted by an actuator on the enclosure shell; the transmitted signal is received by accelerometers mounted on the shell; the useful signal corresponding to a liquid borne path is monitored since gas presence would cause attenuation. An experimental validation and outlines of an industrial application are presented.

\section{Introduction}

Pour la surveillance de nombreux procédés industriels, il est nécessaire de detecter la présence de bulles de gaz dans un liquide contenu par une enceinte métallique. Outre les techniques de détection chimique, il est souvent souhaitable d'utiliser une méthode acoustique. De nombreuses techniques passives, basées sur l'écoute du bruit de la fuite ou de la génération de bulles ont été étudiées [1, $2,3]$.

Mais ces techniques sont tributaires du bruit de fond et peuvent nécessiter un traitement du signal poussé pour des environnements bruyants. Pour éliminer ces inconvénients, nous proposons une méthode active fondée sur la détection de 1'atténuation acoustique créée par la présence d'un nuage de bulles entre un émetteur et un récepteur placés sur l'enceinte. Par rapport aux techniques de mesures Doppler basées sur la rétrodiffusion [4], cette technique est moins sensible aux effets dues à la présence d'une coque métallique.

Dans un premier temps nous allons exposer les principes de la méthode, puis nous présenterons une validation expérimentale dans une situation proche de situations industrielles et finalement nous décrirons une application pratique possible.

\section{Principe de fonctionnement}

I'atténuation acoustique par des bulles de gaz [5] est très importante comparativement à leur taille du fait des phénomènes de résonances dont elle sont le siège. La fréquence propre d'une bulle de rayon a vaut approximativement :

$$
\mathrm{f}=\frac{1}{2 \pi a}\left[\frac{3 \gamma \mathrm{P}}{\rho}\right]^{1 / 2}
$$

où $\gamma$ est le rapport des chaleurs spécifiques du gaz, $\mathrm{P}$ la pression hydrostatique et $\rho$ la masse volumique du liquide. 
Par exemple, pour des bulles d'air dans de l'eau à la pression atmosphérique, la ganme 10-100 kHz correspond à des rayons de bulles résonnantes entre 325 et $32,5 \mu \mathrm{m}$. De plus l'analyse des courbes d'atténuation montre que pour un nuage de bulles, l'atténuation est importante même pour des fréquences éloignées de quelques octaves de la fréquence de résonance moyenne.

Le procédé de détection active [6] que nous avons développé met en valeur ce phénomène de forte absorption. Il consiste à :

- émettre à l'aide d'un actuateur fixé à l'enceinte des trains d'ondes ultrasonores ( au delà de la fréquence de coïncidence des oncies de flexion dans la plaque et des ondes acoustique dans le liquide) ;

- recevoir à l'aide d'accéleromètres disposés sur l'enceinte le signal transmis au travers du système couplé structure - phase liquide ;

- sélectionner, à l'aide de fenêtres temporelles, dans le signal reçu, la partie "utile" correspondant à des transferts à travers la phase liquide ;

- surveiller ce signal utile afin d'y détecter I'amortissement éventuel caractéristique de la présence d'un nuage de bulles.

Le traitement du signal que nous utilisons est très simple : le signal reçu est moyenné quadratiquement (rms). Sur ce signal moyenné, on acquiert le niveau obtenu pour un ou plusieurs délais synchronisés par rapport à l'émission, correspondant à des chemins de transfert pouvant etre perturbés par l'apparition de bulles. Ce niveau est suivi pour chaque train d'ondes et son atténuation indiquera la présence de gaz. (cf Fig 1 )

A titre d'exemple, voici les paramètres d'un des essais que nous avons menés :

$\begin{array}{ll}\text { Bande de fréquence d'émission : } & 20-100 \mathrm{kHz} \\ \text { Longueur du train d'ondes : } & 200 \mu \mathrm{s} \\ \text { Périodicité des trains d'ondes : } 100 \mathrm{~ms}(10 \mathrm{~Hz}) & 0,5 \mathrm{~m} \\ \text { Distance émetteur-récepteur : } & 4,8 \mathrm{~ms} \\ \text { Retard : } & 90 \mu \mathrm{s} \\ \text { Durée d'intégration rms : } & \end{array}$

\section{Validation expérimentale}

Nous avons instrumenté une enceinte de $59 \mathrm{~cm}$ de diamètre, $4 \mathrm{~m}$ de haut et de $30 \mathrm{~mm}$ d'épaisseur remplie de sodium liquide (densité $\approx 800 \mathrm{~kg} / \mathrm{m}^{3}$, célérité $\approx$ $2400 \mathrm{~m} / \mathrm{s}$ ) d'un accéléromètre réciproque servant d'émetteur et d'accéléromètres servant de récepteurs (Figure 2). I'émission était un signal impulsionnel large bande ("pseudo-Dirac") générant une accélération de l'ordre de $1 \mathrm{~g}$ au niveau du récepteur. Une injection d'eau engendrant par réaction chimique de l'hydrogène a été pratiquée avec un débit de $5 \mathrm{~g} / \mathrm{s}$ créant un nuage de gaz d'environ 4 litres au bout de 2 secondes, soit un taux de gaz rapporté au volume de l'enceinte de $4.10^{-3}$.

Les caractéristiques de traitement du signal utilisés dans ce cas sont ceux du tableau ci-dessus. La figure 3 présente le niveau rms reçu par un capteur diamétralement opposé à l'émetteur et situé sur le même plan. Il s'agit d'un zoom autour du "pic" à 4,8 ms pour lequel on a superposé les valeurs mesurées au repos (pointillés) et au bout de 2 secondes d'injection (trait plein).

On note la présence de nombreux pics qui ne sont pas aisément attribuable à un chemin de transfert du fait des multiples voies possibles. L'effet de l'injection est très net sur le pic à $4,8 \mathrm{~ms}^{(1)}$ : on observe une très forte diminution du niveau de la transmission. Par contre pour d'autre retards, on ne remarque aucun effet

(1) Ce retard est largement supérieur au temps de parcours du trajet direct $(0,2 \mathrm{~ms})$ et permet de ce fait une meilleure couverture spatiale de l'enceinte grace aux nombreuses réflexions considérées . 
notable, la transmission solidienne étant peu perturbée par l'injection de gaz.

Actuellement, nous devons déterminer expérimentalement à l'aide d'injections (soit sur l'installation, soit en similitude) les pics de transmission à surveiller, mais des travaux sont en cours pour pouvoir les déterminer sans devoir recourir à ces essais.

\section{Application Industrielle}

La méthode qui vient d'être décrite brièvement est adaptable à un grand nombre de procédés industriels (suivi de procédé chimique, détection de gaz dans les batteries, détection d'ébullition...) . Nous l'avons développée en vue de la détection de présence d'hydrogène dans les générateurs de vapeurs de réacteurs à neutrons rapides refroidis au sodium [6].

Pour le projet European Fast Reactor, nous considérons des cylindres de $30 \mathrm{~m}$ de haut et de $1,5 \mathrm{~m}$ de rayon $\left(50 \mathrm{~m}^{3}\right)$ dans lequel s'effectuent les transferts thermiques entre les circuits de sodium secondaires et d'eau/vapeur. Notre objectif est de détecter une fuite générant quelques litres de gaz en quelques secondes avec une probabilité de non-détection de $10^{-3}$ par fuite et un taux de fausse alarme inférieur à une pour 10 ans.

Pour atteindre ce but, nous mettons au point un système utilisant à la fois la technique active et une écoute passive utilisant les mêmes récepteurs. . Les interférences entre les deux techniques sont évitées en éliminant pour le traitement passif d'une part la bande de fréquence d'émission (dans ce cas le signal d'émission sera à support fréquentiel étroit) d'autre part la fenêtre temporelle correspondant à cette dernière.

Du fait de la température élevée des parois et de la présence d'un calorifuge, les capteurs sont montés au bout d'un guide d'onde cylindrique soudé à la virole. L'ordre de grandeur du nombre de transducteurs est autour de cinquante.

\section{Conclusion}

Nous avons présenté un procédé de détection acoustique de la présence de gaz dans une enceinte métallique remplie de liquide. Ses principaux avantages sont :

- la rapidité de detection (quelques secondes);

- l'absence de capteurs à l'intérieur de l'enceinte et, donc la possibilité de surveiller aisément des fluides corrosifs ou dans des situations à température ou à pression élevée ;

- le peu d'influence du bruit ambiant.

Une validation expérimentale et les grandes lignes d'une application à la détection de présence d'hydrogène dans les générateurs de vapeur de réacteurs à neutrons rapides ont été présentées.

\section{Remerciements}

Ces travaux ont été effectués dans le cadre de l'accord de coopération en R\&D pour le Réacteur Rapide Européen (European Fast Reactor). Les essais présentés ont été réalisés sur la boucle ASB d'Interatom $\mathrm{GmbH}$ à Bensberg, Allemagne que nous remercions pour leur aide.

Réferences

[1] M.Strasberg Gas Bubbles as Source of Sound in Liquids J.A.S.A. 28,20 (1956)

[2] T.N. Claytor, D.A. Greene Measurements of Acoustic Noise Produced by Steam Injections into Sodium, Trans. ANS, 23 p 502-503 (1982)

[3] M.Brunet et al. Water leak Detection in Steam Generator of Superphenix, Spec 
Meeting on Reactor Noise (SMORN V), Munich 1987

[4] R.Y. Nishi Ultrasonic Detection of bubbles with doppler flow measurements, Ultrasonics 10 p 173-179 (7/1972)

[5] C.S. Clay, H. Medwin Acoustical Oceanography, Wiley-Interscience, New York 1977

[6] P.Garnaud, R.Demarais Procédé de détection par ultrasons de l'apparition d'une phase gazeuse dans une phase liquide contenue dans une enceinte, Brevet français $n^{\circ} 9000934(26 / 1 / 90)$

[7] D.Blanc, D.Villani, J. Ford Toward an active acoustic detection system on EFR AIEA Spec. Meeting on Acoust. Detection of in sodium water leaks, Aix en Provence 1990, editor : JPh. Girard,CEA Cadarache.

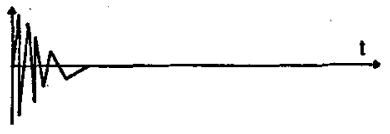

Signal d'Emission
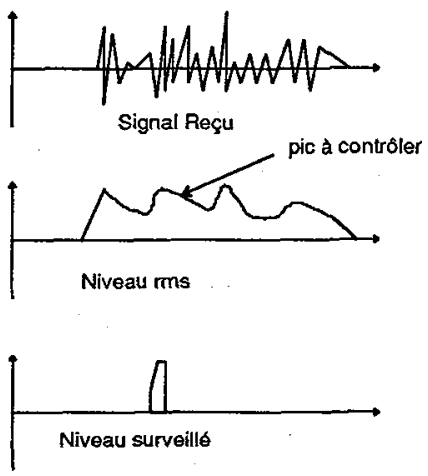

Figure 1: Signaux considérés

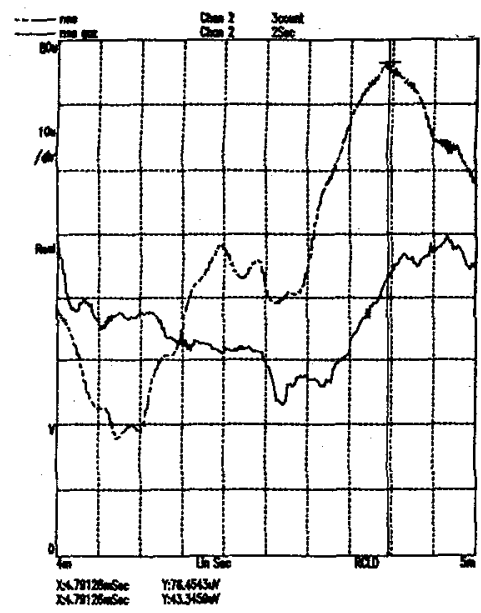

Figure 3:

Signaux recus avec et sans injection

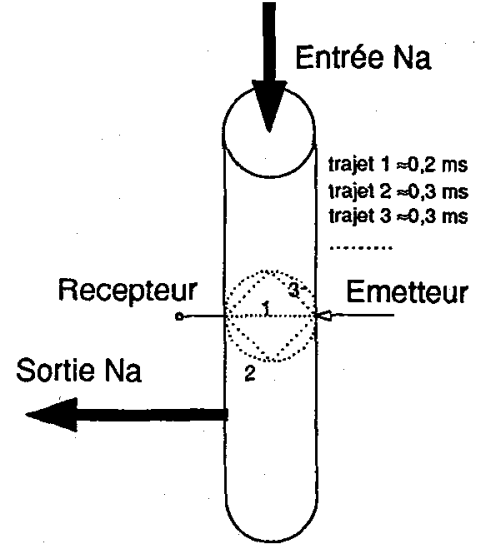

Enceinte

d'essai

Figure 2 : Dispositif Expérimental

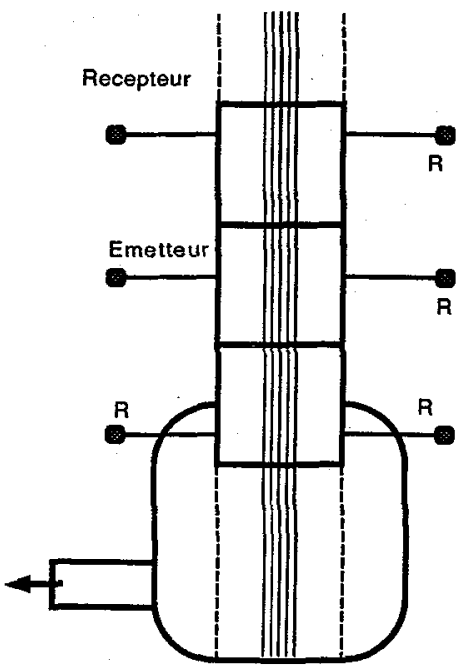

Figure 4 :

Implantation Industrielle Envisagée 